\title{
Boson stars in massive dilatonic gravity
}

\author{
Plamen P. Fiziev ${ }^{1}$, Stoytcho S. Yazadjiev ${ }^{1}$ \\ ${ }^{1}$ Department of Theoretical Physics, Faculty of Physics, Sofia University, Bulgaria \\ Todor L. Boyadjiev ${ }^{2}$ and Michail D. Todorov ${ }^{3}$ \\ ${ }^{2}$ Faculty of Mathematics and Computer Science, Sofia University, Bulgaria \\ ${ }^{3}$ Institute of Applied Mathematics and Computer Science, Technical University, Sofia, Bulgaria
}

\begin{abstract}
We study equilibrium configurations of boson stars in the framework of a class scalar-tensor theories of gravity with massive gravitational scalar (dilaton). In particular we investigate the influence of the mass of the dilaton on the boson star structure. We find that the masses of the boson stars in presence of dilaton are close to those in general relativity and they are sensitive to the ratio of the boson mass to the dilaton mass within a typical few percent. It turns out also that the boson star structure is mainly sensitive to the mass term of the dilaton potential rather to the exact form of the potential .
\end{abstract}

PACS number(s): 04.50.+h, 04.40.Dg, 95.35.+d

\section{INTRODUCTION}

Boson stars are gravitationally bound macroscopic quantum states made up of scalar bosons. They differ from the fermionic stars in that they are only prevented from collapsing gravitationally by the Heisenberg uncertainty principle. Boson stars were first considered by Kaup [1] and then by Ruffini and Bonazola [2]. They found that the boson stars as described by non-interacting massive, complex scalar field had masses of the order of $\mathcal{M} \approx \frac{\mathcal{M}_{P l}^{2}}{m_{B}}$, where $\mathcal{M}_{P l}$ is the Planck mass and $m_{B}$ is the boson mass. In a latter work Colpi, Shapiro and Wasserman [3] analyzed the consequence of switching on a quartic self-interaction for the boson field. This results in a drastic increase of the mass of the boson star, which even for small values of the coupling constant turns out to be of the order of the Chandrasekhar's mass when the boson mass is similar to a proton mass. Thus, the boson stars arise as possible candidates for non-baryonic dark matter in the universe. Although we still have no astrophysical evidences for their existence, the boson stars are good model to learn more about the nature of strong gravitational fields not only in general relativity but also in the other theories of gravity.

The most natural and promising generalizations of Einstein's general relativity are scalar-tensor theories of gravity. The most studied class of such theories are scalar-tensor theories with massless gravitational scalar field including the Brans-Dicke theory as a special case. It should be noted that an interesting specific class of scalar-tensor theories with dilaton field arise from the low energy limit of string theory, which confirms their importance for current physics.

Boson stars in the framework of scalar-tensor theories with massless gravitational scalar have been studied widely, too. The first scalar-tensor model of a boson star was studied by Gundersen and Jensen [4], who considered
Brans-Dicke theory with $\omega_{B D}=6$. Their work was generalized by Torres [5], who studied boson stars in scalartensor theories with nonconstant $\omega_{B D}(\Phi)$. The conclusion is that boson stars can exist in any scalar-tensor theory of gravity with massless gravitational scalar, as the masses of the boson stars are always smaller than in the general relativistic case irrespective of the coupling functions $\omega_{B D}(\Phi)$. We should mention also the interesting paper by Z. Tao and X. Xue [6] where boson stars are studied in the framework of a scalar-tensor theory with a coupling between the dilaton and the mass term of the boson field.

More recently boson stars in scalar-tensor theories have been studied in the papers by Torres et al [7], 9] and in the paper by Comer and Shinkai 8. In [7] boson stars have been studied in connection with so-called gravitational memory [10], while their stability through cosmic history has examined in [8] and [9]. The dynamical evolution of boson stars has been investigated in the paper by Balakrishna and Shinkai [11. Charged boson stars in scalar-tensor gravity with massless gravitational scalar have been studied in [12].

As we already mentioned boson stars in scalar-tensor theories have been studied only in the case of scalartensor theories of gravity with massless gravitational scalar. From a field-theoretical point of view it is to be expected that the scalar part of the gravitational field is massive and leads to a force with a finite range. It also should be noted that there is no symmetry which forbids a mass term, or a more general potential term for the gravitational scalar. Moreover, if string theory and it's low energy limit are relevant to the real world then the dilaton must be massive. Unfortunately, our current understanding of how the dilaton acquires mass is rather primitive and it's tied to our lack of understanding of supersymmetry breaking. Since we don't have a definite model for dilaton mass generation the mass of the dila- 
ton and the form of its potential are completely unknown from theoretical point of view.

The purpose of the present paper is to study boson stars in the framework of some class of scalar-tensor theories of gravity with massive gravitational scalar. In particular we study the possible influence of the mass and more generally of the potential of gravitational scalar on the equilibrium configurations and stability of the boson stars. The obtained results, however, are typical for wider classes of scalar-tensor theories with massive dilaton not only for the class we consider.

\section{A SCALAR-TENSOR THEORY OF GRAVITY WITH MASSIVE GRAVITATIONAL SCALAR}

The most general scalar-tensor theory of gravity including such a term is described by the following action

$$
\begin{array}{r}
\tilde{\mathcal{A}}=-\frac{1}{16 \pi G_{*}} \int d^{4} x \sqrt{-\tilde{g}}\left(\Phi \tilde{R}-h(\Phi) \tilde{g}^{\mu \nu} \partial_{\mu} \Phi \partial_{\nu} \Phi\right. \\
+\tilde{U}(\Phi))+\mathcal{A}_{\text {matter }}\left(\Psi_{\text {matter }}, \tilde{g}_{\mu \nu}\right)
\end{array}
$$

where $\tilde{g}_{\mu \nu}$ is the space-time metric, $\tilde{R}$ is the Ricci scalar curvature with respect to the space-time metric. The functions $h(\Phi)$ and $\tilde{U}(\Phi)$ are, in general, arbitrary differentiable functions of the gravitational scalar $\Phi$. A new method for determination of these functions using astrophysical observations is developed in the recent articles [13], but at present we have no good enough data to make use of this method. The last term in (11) denotes the action of the matter, which is a functional of the matter variables, collectively denoted by $\Psi_{\text {matter }}$, and of the space-time metric $\tilde{g}_{\mu \nu}$.

For our purpose in this article the action (11) is too general. That's why we restrict ourselves to a subclass of scalar-tensor theories which don't include explicitly a kinetic term for the gravitational scalar in the action (i.e. with function $h(\Phi) \equiv 0$ ). In particular we consider scalar-tensor theories described by the action

$$
\begin{aligned}
\tilde{A}=\frac{-1}{16 \pi G_{*}} \int & d^{4} x \sqrt{-\tilde{g}}(\Phi \tilde{R}+\tilde{U}(\Phi)) \\
& +\mathcal{A}_{\text {matter }}\left(\Psi_{\text {matter }}, \tilde{g}_{\mu \nu}\right) .
\end{aligned}
$$

Such scalar-tensor theory with only one unknown function $\tilde{U}(\Phi)$ is much more definite. It has been considered at first by O'Hanlon [14 in connection with Fujii's theory of massive dilaton [15]. It may be considered also as a part of more general theories of gravity like $(4+1)$ dimensional Kaluza-Klein model described by Fujii in [16], or model of gravity with violated local conformal symmetry in affine-connected spaces which probably may be related with string theory [17]. A recent development of model of dilatonic gravity with action (2) related with newest astrophysical data on cosmological constant problem may be found in [18]. In addition there it was shown that this model is consistent with extremely high precision with all known data in solar system gravitational experiments if one chooses properly the potential $\tilde{U}(\Phi)$. Under proper normalization of this potential solar system end Earth surface experiments actually give restrictions only on the mass term in the potential $U(\Phi)$.

The corresponding field equations are

$$
\begin{gathered}
\tilde{G}_{\mu \nu}=\frac{\kappa_{*}}{\Phi} \tilde{T}_{\mu \nu}+\frac{1}{\Phi}\left(\tilde{\nabla}_{\mu} \tilde{\nabla}_{\nu} \Phi-\tilde{g}_{\mu \nu} \tilde{\nabla}_{\rho} \tilde{\nabla}^{\rho} \Phi\right)+\frac{1}{2} \frac{\tilde{U}(\Phi)}{\Phi} \tilde{g}_{\mu \nu} \\
\tilde{\nabla}_{\rho} \tilde{\nabla}^{\rho} \Phi+\frac{1}{3}\left(\Phi \frac{d \tilde{U}(\Phi)}{d \Phi}-2 \tilde{U}(\Phi)\right)=\frac{\kappa_{*}}{3} \tilde{T}
\end{gathered}
$$

For the treatment of the local Earth surface, astrophysical and astronomical problems in star systems (but not for cosmological problems in the scales of universe) we demand that a weak-field approximation should be possible assuming in addition that the space-time is asymptotically flat. Then in weak-field approximation we may write

$$
\tilde{g}_{\mu \nu}=\eta_{\mu \nu}+\delta \tilde{h}_{\mu \nu}, \quad \Phi=\Phi_{\infty}+\delta \Phi
$$

where $\eta_{\mu \nu}$ is the flat space-time metric, $\Phi_{\infty}$ is the background value of the $\Phi$, and $\delta h_{\mu \nu}$ and $\delta \Phi$ are small perturbations due to the local masses. Consistency of the field equations then requires that

$$
\tilde{U}\left(\Phi_{\infty}\right)=\frac{d \tilde{U}}{d \Phi}\left(\Phi_{\infty}\right)=0 .
$$

Using now the consistency conditions (5) we obtain that the mass of the gravitational scalar $\Phi$ is

$$
m^{2}=\frac{\Phi_{\infty}}{3} \frac{d^{2} \tilde{U}}{d \Phi^{2}}\left(\Phi_{\infty}\right)
$$

Further on, without loss of generality we take $\Phi_{\infty}=1$. In this case, taking into account that the range of the gravitational scalar is finite we obtain that the bare gravitational constant $G_{*}$ coincides with the background physical gravitational constant.

The PPN formalism for scalar-tensor theories of gravity with a massive gravitational scalar has been developed by Helbig [19], who has also calculated the basic gravitational effects as perihelion shift and so on. Using Helbig's results and the experimental data published in [20] and [21] we obtain that the scalar-tensor theory we consider here is consistent with the experiments when the Compton length of the gravitational scalar $\Phi$ is less than $2 \mathrm{~mm}$, or equivalently when the mass of the gravitational scalar satisfies the constraint $m \geq 10^{-4} \mathrm{eV} 18$.

So far our considerations have been made in the Jordan frame. For some purposes, in particular for numerical calculations, the scalar-tensor theories are better formulated in the conformal Einstein frame. The metric in the Einstein frame is given by the conformal transformation 


$$
g_{\mu \nu}=\Phi \tilde{g}_{\mu \nu} .
$$

It should be stressed that it's the Jordan frame which is the physical frame while the Einstein frame is a convenient mathematical tool. Including also the dilaton field $\varphi$ via the formula $\Phi=\exp (-2 \alpha \varphi)$, where $\alpha=\frac{1}{\sqrt{3}}$, the action (2) written in terms of $g_{\mu \nu}$ and $\varphi$ reads

$$
\begin{array}{r}
\mathcal{A}=-\frac{1}{16 \pi G_{*}} \int d^{4} x \sqrt{-g}\left(R-2 g^{\mu \nu} \partial_{\mu} \varphi \partial_{\nu} \varphi+U(\varphi)\right) \\
+\mathcal{A}_{\text {matter }}\left(\Psi_{\text {matter }}, A^{2}(\varphi) g_{\mu \nu}\right) .
\end{array}
$$

Here $R$ is the Ricci scalar curvature with respect to the metric $g_{\mu \nu}, U(\varphi)=A^{4}(\varphi) \tilde{U}(\Phi(\varphi))$ is the potential of the dilaton field $\varphi$ and $A^{2}(\varphi)=\Phi^{-1}(\varphi)$. It should be noted that the convention $\Phi_{\infty}=1$ in terms of the dilaton field writes $\varphi_{\infty}=0$. As must be expected the dilaton potential $U(\varphi)$ satisfies the conditions $U\left(\varphi_{\infty}\right)=\frac{d U(\varphi)}{d \varphi}\left(\varphi_{\infty}\right)=0$ and the mass of the dilaton coincides with the mass of the gravitational scalar $\Phi$ which gives another equivalent representation of the same physical object.

\section{BOSON STAR MODEL}

We take the matter action to be the action of complex, massive and self-interacting scalar field $\Psi$ which in the physical Jordan frame has the form

$$
\mathcal{A}_{\text {matter }}=\int d^{4} x \sqrt{-\tilde{g}}\left(\frac{1}{2} \tilde{g}^{\mu \nu} \partial_{\mu} \Psi^{+} \partial_{\nu} \Psi-W\left(\Psi^{+} \Psi\right)\right)
$$

where $W\left(\Psi^{+} \Psi\right)=\frac{1}{2} m_{B}^{2} \Psi^{+} \Psi+\frac{1}{4} \hat{\Lambda}\left(\Psi^{+} \Psi\right)^{2}$ is the potential of the boson field. Then the action of the gravity and matter in Einstein frame is

$$
\begin{gathered}
\mathcal{A}=-\frac{1}{16 \pi G_{*}} \int d^{4} x \sqrt{-g}\left(R-2 g^{\mu \nu} \partial_{\mu} \varphi \partial_{\nu} \varphi+U(\varphi)\right) \\
+\int d^{4} x \sqrt{-g}\left(\frac{1}{2} A^{2}(\varphi) g^{\mu \nu} \partial_{\mu} \Psi^{+} \partial_{\nu} \Psi\right. \\
\left.-A^{4}(\varphi) W\left(\Psi^{+} \Psi\right)\right)
\end{gathered}
$$

Varying the action (10) with respect to $g_{\mu \nu}, \varphi, \Psi$ and $\Psi^{+}$we obtain the field equations

$$
\begin{array}{r}
G_{\mu \nu}=\kappa_{*} T_{\mu \nu}+2 \partial_{\mu} \varphi \partial_{\nu} \varphi-g_{\mu \nu} \partial_{\rho} \varphi \partial^{\rho} \varphi+\frac{1}{2} U(\varphi) g_{\mu \nu}, \\
\nabla_{\rho} \nabla^{\rho} \varphi+\frac{1}{4} U^{\prime}(\varphi)=-\frac{\alpha}{2} \kappa_{*} T, \\
\nabla_{\rho} \nabla^{\rho} \Psi+2 \alpha \partial_{\rho} \varphi \partial^{\rho} \Psi=-2 A^{2}(\varphi) \frac{d W\left(\Psi^{+} \Psi\right)}{d \Psi^{+}}, \\
\nabla_{\rho} \nabla^{\rho} \Psi^{+}+2 \alpha \partial_{\rho} \varphi \partial^{\rho} \Psi^{+}=-2 A^{2}(\varphi) \frac{d W\left(\Psi^{+} \Psi\right)}{d \Psi}
\end{array}
$$

where $\nabla_{\rho}$ is the Levi-Civita connection with respect to the metric $g_{\mu \nu}, T_{\mu \nu}$ is the Einstein frame energymomentum tensor of the boson filed $\Psi$ and $T$ is its trace. The Einstein frame energy-momentum tensor $T_{\mu \nu}$ is given by

$$
\begin{gathered}
T_{\mu \nu}=\frac{1}{2} A^{2}(\varphi)\left(\partial_{\mu} \Psi^{+} \partial_{\nu} \Psi+\partial_{\mu} \Psi \partial_{\nu} \Psi^{+}\right)- \\
\frac{1}{2} A^{2}(\varphi)\left(\partial_{\rho} \Psi^{+} \partial^{\rho} \Psi-2 A^{2}(\varphi) W\left(\Psi^{+} \Psi\right)\right) g_{\mu \nu}
\end{gathered}
$$

We consider a static and spherically symmetric boson star. Then the metric $g_{\mu \nu}$ can be written in the standard form

$$
d s^{2}=e^{\nu(\rho)} d t^{2}-e^{\lambda(\rho)} d \rho^{2}-\rho^{2}\left(d \theta^{2}+\sin ^{2}(\theta) d \phi^{2}\right) .
$$

We also demand a spherically-symmetric form for the boson field and we adopt a form consistent with the static metric:

$$
\Psi=\hat{\sigma}(\rho) e^{i \omega t}
$$

Using the metric (13) and the equation defining the form of the boson field together with the Einstein frame energy-momentum tensor (12) in the field equations (11) we get the equations for the structure of the boson star. Before we explicitly write them we are going to introduce a dimensionless radial coordinate by

$$
r=m_{B} \rho .
$$

From now on, a prime will denote a differentiation with respect to the dimensionless coordinate $r$. We also define other dimensionless quantities by

$$
\Omega=\frac{\omega}{m_{B}}, \sigma=\sqrt{\kappa_{*}} \hat{\sigma}, \Lambda=\frac{\hat{\Lambda}}{\kappa_{*} m_{B}}, \gamma=\frac{m}{m_{B}}
$$

and dimensionless potential $V(\varphi)$ given by

$$
U(\varphi)=m^{2} V(\varphi)
$$

With all these definitions, the equations of the structure of the boson star in Einstein frame reduce to the following

$$
\begin{array}{r}
\lambda^{\prime}=\frac{1-e^{\lambda}}{r}+r e^{\lambda} \mathcal{T}_{0}^{0}+r \varphi^{2}+\frac{1}{2} \gamma^{2} r e^{\lambda} V(\varphi), \\
\nu^{\prime}=\frac{e^{\lambda}-1}{r}-r e^{\lambda} \mathcal{T}_{1}^{1}+r \varphi^{\prime 2}-\frac{1}{2} \gamma^{2} r e^{\lambda} V(\varphi), \\
\nu^{\prime \prime}=\frac{-\nu^{\prime 2}+\lambda^{\prime}-\nu^{\prime}+\lambda^{\prime} \nu^{\prime}}{2}-2 e^{\lambda} \mathcal{T}_{2}^{2}-2 \varphi^{\prime 2}-\gamma^{2} e^{\lambda} V(\varphi), \\
\varphi^{\prime \prime}=-\left(\frac{\nu^{\prime}-\lambda^{\prime}}{2}+\frac{2}{r}\right) \varphi^{\prime}+\frac{1}{4} \gamma^{2} e^{\lambda} \frac{d V(\varphi)}{d \varphi}+\frac{\alpha}{2} \mathcal{T} e^{\lambda}, \\
\sigma^{\prime \prime}=-\left(\frac{\nu^{\prime}-\lambda^{\prime}}{2}+\frac{2}{r}\right) \sigma^{\prime}-\Omega^{2} e^{\lambda-\nu} \sigma-2 \alpha \varphi^{\prime} \sigma^{\prime}+ \\
2 A^{2}(\varphi) e^{\lambda} \frac{d W\left(\sigma^{2}\right)}{d \sigma^{2}} \sigma .
\end{array}
$$

Here $\mathcal{T}_{\nu}^{\mu}=\frac{\kappa_{*}}{m_{B}^{2}} T_{\nu}^{\mu}$ is the dimensionless Einstein frame energy-momentum tensor and $\mathcal{T}$ is its trace. In explicit form we have 


$$
\begin{aligned}
\mathcal{T}_{0}^{0} & =\frac{1}{2} \Omega^{2} A^{2}(\varphi) e^{-\nu} \sigma^{2}+\frac{1}{2} A^{2}(\varphi) e^{-\lambda}{\sigma^{\prime}}^{2}+A^{4}(\varphi) W\left(\sigma^{2}\right), \\
\mathcal{T}_{1}^{1} & =-\frac{1}{2} \Omega^{2} A^{2}(\varphi) e^{-\nu} \sigma^{2}-\frac{1}{2} A^{2}(\varphi) e^{-\lambda}{\sigma^{\prime}}^{2}+A^{4}(\varphi) W\left(\sigma^{2}\right), \\
\mathcal{T}_{2}^{2} & =-\frac{1}{2} \Omega^{2} A^{2}(\varphi) e^{-\nu} \sigma^{2}+\frac{1}{2} A^{2}(\varphi) e^{-\lambda}{\sigma^{\prime}}^{2}+A^{4}(\varphi) W\left(\sigma^{2}\right), \\
\mathcal{T} & =-\Omega^{2} A^{2}(\varphi) e^{-\nu} \sigma^{2}+A^{2}(\varphi) e^{-\lambda}{\sigma^{\prime}}^{2}+4 A^{4}(\varphi) W\left(\sigma^{2}\right) .
\end{aligned}
$$

the only quantity which changes under transition to Jordan frame is $\nu$. The non-singularity at the center and the asymptotic flatness in Jordan frame require correspondingly that $\tilde{\nu}^{\prime}(0)=0$ and $\tilde{\nu}(\infty)=0$. Taking into account that $\tilde{\nu}=\nu+2 \alpha \varphi$ and Einstein frame boundary conditions for the dilaton field $\varphi$ we obtain that Jordan frame boundary conditions for $\tilde{\nu}$ are satisfied if and only if $\nu^{\prime}(0)=\nu(\infty)=0$.

\section{CONSERVED QUANTITIES}

The action of the boson field is $U(1)$-invariant under the global gauge transformations $\Psi \rightarrow e^{i a} \Psi$, where $a$ is a constant. This global $U(1)$-symmetry gives rise to the following conserved current in Jordan frame

$$
\begin{gathered}
\nu^{\prime \prime}=-\frac{\nu^{\prime}}{r}+\left(-\frac{\nu^{\prime}}{r}+\mathcal{T}_{0}^{0}-\mathcal{T}_{1}^{1}+2 \mathcal{T}_{2}^{2}-\gamma^{2} V(\varphi)+\right. \\
\left.\frac{\nu^{\prime} r}{2}\left(\mathcal{T}_{0}^{0}+\mathcal{T}_{1}^{1}+\gamma^{2} V(\varphi)\right)\right) e^{\lambda} \\
\varphi^{\prime \prime}=-\frac{\varphi^{\prime}}{r}+\left(-\frac{\varphi^{\prime}}{r}+\frac{\alpha}{2} \mathcal{T}+\frac{1}{4} \gamma^{2} \frac{d V(\varphi)}{d \varphi}+\right. \\
\left.\frac{\varphi^{\prime} r}{2}\left(\mathcal{T}_{0}^{0}+\mathcal{T}_{1}^{1}+\gamma^{2} V(\varphi)\right)\right) e^{\lambda} \\
\sigma^{\prime \prime}=-\frac{\sigma^{\prime}}{r}-2 \alpha \varphi^{\prime} \sigma^{\prime}+\left(-\frac{\sigma^{\prime}}{r}+2 A^{2}(\varphi) \frac{d W\left(\sigma^{2}\right)}{d \sigma^{2}} \sigma-\right. \\
\left.\Omega^{2} e^{-\nu} \sigma+\frac{\sigma^{\prime} r}{2}\left(\mathcal{T}_{0}^{0}+\mathcal{T}_{1}^{1}+\gamma^{2} V(\varphi)\right)\right) e^{\lambda}
\end{gathered}
$$

where $e^{\lambda}$ is given by

$e^{\lambda}=\frac{1+r \nu^{\prime}-r^{2}{\varphi^{\prime}}^{2}-\frac{1}{2} A^{2}(\varphi) r^{2}{\sigma^{\prime}}^{2}}{1-r^{2}\left(\frac{1}{2} \gamma^{2} V(\varphi)-\frac{1}{2} \Omega^{2} A^{2}(\varphi) e^{-\nu} \sigma^{2}+A^{4}(\varphi) W\left(\sigma^{2}\right)\right)}$.

We solve numerically the system (20). The boundary conditions for the system are the following. We demand asymptotic flatness which means that $\nu(\infty)=0$. The non-singularity of the $\nu$ at the center of the star requires that $\nu^{\prime}(0)=0$. Concerning the dilaton $\varphi$, the non-singularity at the center implies $\varphi^{\prime}(0)=0$, while at infinity we must have $\varphi(\infty)=0$ as required by the asymptotic flatness. Non-singularity of the boson field at the center implies $\sigma^{\prime}(0)=0$ and the asymptotic flatness requires that $\sigma(\infty)=0$. To complete the nonlinear eigenvalue problem for $\Omega$ we also must give the central value of the boson field $\sigma(0)=\sigma_{c}$.

Here we must make some comments. We have imposed the boundary conditions in Einstein frame. But what is important is the non-singularity and asymptotic flatness in the physical Jordan frame. However, it's not difficult to see that all Einstein frame boundary conditions phrased in Jordan frame remain the same. Indeed,

$$
\tilde{J}^{\mu}=\frac{i}{2} \sqrt{-\tilde{g}} \tilde{g}^{\mu \nu}\left(\Psi \partial_{\nu} \Psi^{+}-\Psi^{+} \partial_{\nu} \Psi\right) .
$$

The same current written in terms of the Einstein frame metric is

$$
J^{\mu}=\frac{i}{2} A^{2}(\varphi) \sqrt{-g} g^{\mu \nu}\left(\Psi \partial_{\nu} \Psi^{+}-\Psi^{+} \partial_{\nu} \Psi\right) .
$$

The conserved current leads to conserved charge - the total number of particles making up the star:

$$
N=\int \tilde{J}^{0} d^{3} x
$$

It should be stressed that $N$ is the total number of particles in the physical Jordan frame. Taking into account the explicit form of the metric and of the boson field we obtain

$$
N=\omega \int d^{3} x \sqrt{-\tilde{g}} \tilde{g}^{00} \hat{\sigma}^{2}
$$

In order to calculate numerically the total number of particles we must present the above integral in terms of Einstein frame metric. This can be done easily by presenting the Jordan frame metric as conformally transformed Einstein frame metric. This way we obtain

$\mathcal{M}_{R}=m_{B} N=\left(\frac{\mathcal{M}_{P l}^{2}}{2 m_{B}}\right) \Omega \int_{0}^{\infty} d r r^{2} A^{2}(\varphi) e^{\frac{\lambda-\nu}{2}} \sigma^{2}$

where $\mathcal{M}_{R}$ is the rest mass of the star in the Jordan frame.

The binding energy in Jordan frame is then defined by

$$
\mathcal{E}_{b}=\mathcal{M}-\mathcal{M}_{R}
$$

where $\mathcal{M}$ is the total mass of the star.

In contrast to the general relativity the definition of mass in scalar-tensor theories is subtle. There are three possible mass definitions in Jordan frame, notably the Schwarzschild mass $\mathcal{M}_{S}$ (i.e. ADM mass in Jordan frame), the Kepler mass $\mathcal{M}_{K}$ and the tensor mass $\mathcal{M}_{T}$ 
which is the ADM mass in Einstein frame [22]. These mass definitions are equivalent in general relativity, but they may give different results in scalar-tensor theories of general type. It's the tensor mass which has got physically acceptable properties: The tensor mass has the important property to peak, as a function of the central density at the same location where the particle number (respectively the rest mass) takes its maximum. This property has been proved in the most general scalartensor theory including a potential term for the gravitational scalar (dilaton), see for details [23]. That the tensor mass and particle number peak at the same location results in a cusp in the bifurcation diagram $\mathcal{M}$ (respectively $\mathcal{E}_{b}$ ) versus $\mathcal{M}_{R}$.

All masses we have mentioned are asymptotically measured quantities. Taking into account that in the scalartensor theory we consider the gravitational scalar (dilaton) varies essentially in a finite space-time domain, it's not difficult to see that all masses in our case are identical. That's why all properties proven for the tensor mass are shared also by the others and the mass of the star may be expressed in three different ways as one wishes. We take the expression for the mass of the star in the form of the Schwarzschild mass in Einstein frame, namely

$\mathcal{M}=\left(\frac{\mathcal{M}^{2}{ }_{P l}}{2 m_{B}}\right) \int_{0}^{\infty} d r r^{2}\left(\mathcal{T}_{0}^{0}+e^{-\lambda} \varphi^{\prime 2}+\frac{1}{2} U(\varphi)\right)$.

For numerical purposes we also introduce the following dimensionless masses

$$
M=\mathcal{M} / \frac{\mathcal{M}_{P l}^{2}}{2 m_{B}} \quad, \quad M_{R}=\mathcal{M}_{R} / \frac{\mathcal{M}_{P l}^{2}}{2 m_{B}} .
$$

Therefore the dimensionless binding energy is

$$
E_{b}=M-M_{R}
$$

We also define an effective dimensionless radius of the boson star as:

$$
R=\frac{m_{B}}{N} \int r J^{0} d^{3} x
$$

\section{NUMERICAL RESULTS AND DISCUSSIONS}

To solve the nonlinear ODE's (20) with the corresponding boundary conditions and spectral parameter $\Omega$ we apply the continuous analog of the Newton method [24, [25]. In our case this method leads to a linear systems ODE's with respect to the increments of unknown functions coupling with an algebraic equation for the increment of $\Omega$. The appropriate linear boundary value problems we solve numerically using the spline collocation at the Gaussian points on the irregular mesh condensing to the centre $r=0$ of the star. For details see our work [26.

As we have already mentioned at present we have only some weak experimental constrains on the mass of the

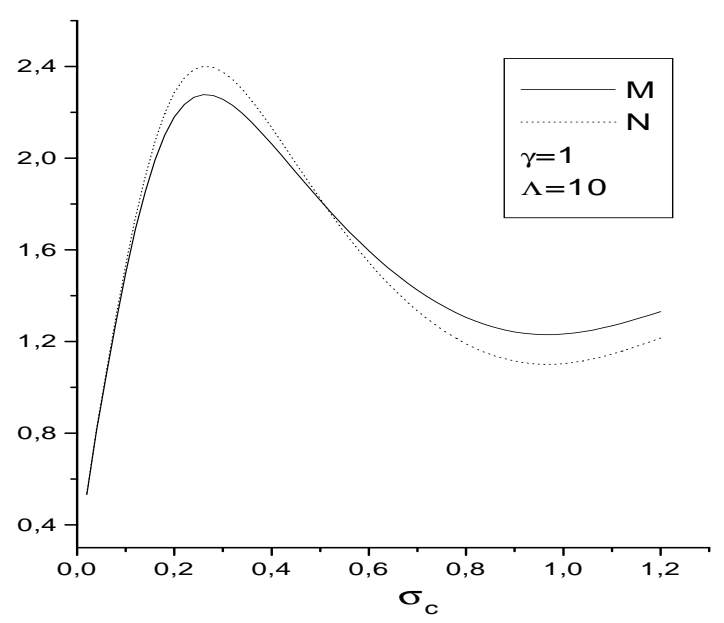

FIG. 1. The dependence of the mass and rest mass of the boson star on the central value $\sigma_{c}$

dilaton and on the form of its potential. A definite theoretical suggestions for them do not exist. That's why we consider first the simplest form for the potential of the gravitational scalar

$$
\tilde{U}(\Phi)=\frac{3}{2} m^{2}(\Phi-1)^{2} .
$$

The corresponding dilaton potential is

$$
U(\varphi)=m^{2} V(\varphi)=\frac{3}{2} m^{2}\left(1-\exp \left(\frac{2 \varphi}{\sqrt{3}}\right)\right)^{2}
$$

The numerical results for the chosen potential are presented in the figures.

Fig.1 presents a configuration diagram which is typical for large variations of the parameters not only for those values presented on the figure.

The dependence of the boson star mass on the central value $\sigma_{c}$ is presented in Fig.2 for three different values of the ratio $\gamma=\frac{m}{m_{B}}$ at fixed value of the parameter $\lambda=10$. It's seen that the curves $M\left(\sigma_{c}\right)$ differ slightly from each other. More detailed investigation for much more values of $\gamma$ shows that the curves tend to the same curve $M\left(\sigma_{c}\right)$ from the general relativistic case for large values of $\gamma(\gamma \approx 10)$. This behaviour is confirmed also by Fig. 3 where the dependence of the boson star mass on the parameter $\gamma$ is shown for $\sigma_{c}=0.1$ and $\Lambda=10$. For small values of $\gamma$ the boson star mass increases when the parameter $\gamma$ increases. Then for larger values of $\gamma$ the mass $M$ tends to a fixed value which is just the general relativistic value. The cause for this behaviour is that the increasing of the parameter $\gamma$ is actually an increasing of the dilaton mass with respect to the boson mass which leads to smaller dilaton range and therefore to a 


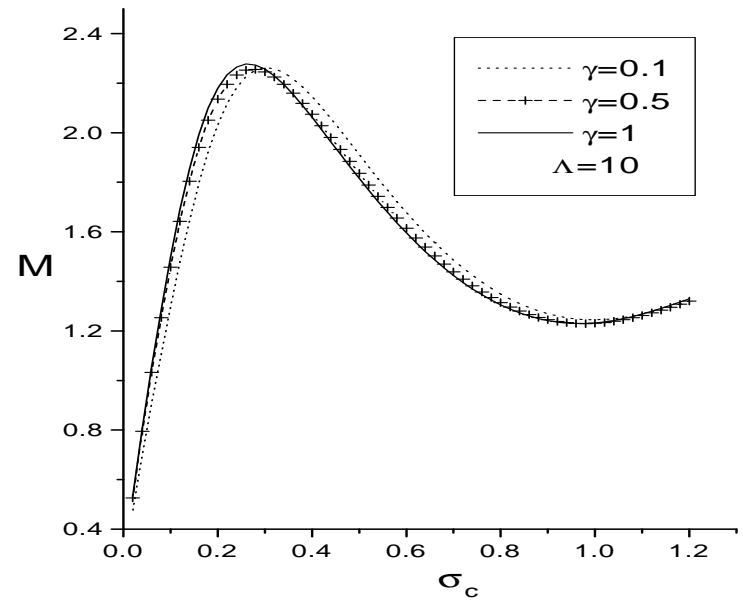

FIG. 2. The dependence of the boson star mass on the central value $\sigma_{c}$ for three different values of the parameter $\gamma$.

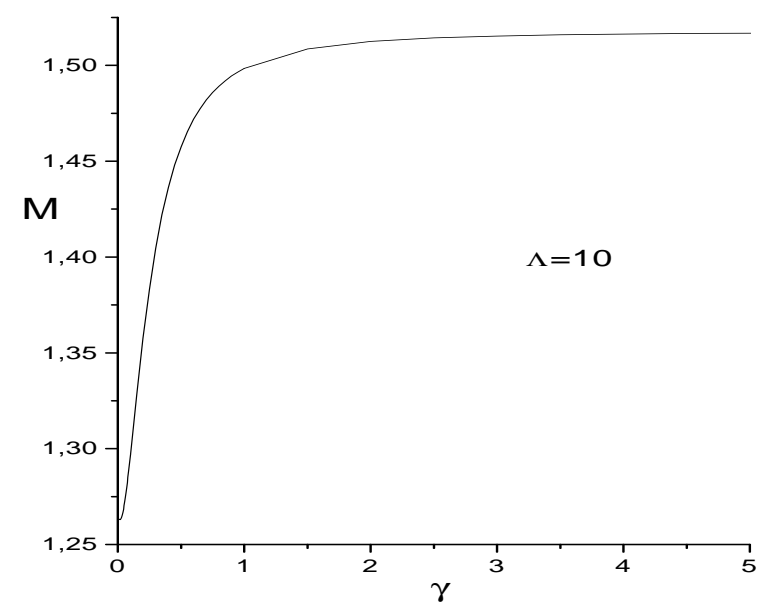

FIG. 3. The dependence of the boson star mass on the parameter $\gamma$ for $\Lambda=10$

suppressing of the dilaton field which means that the general relativity is recovered. The Fig. 3 also shows that the boson stars in the model under consideration always have masses smaller than those in general relativity. The deviations from general relativity are within a typical few percent.

The influence of the dilaton mass, respectively $\gamma$, on the dependence of the boson field on the radial coordinate $r$ is very slightly as one may see from Fig. 4. The dependence $\sigma(r)$ is presented there for three different values of $\gamma$. The three curves are extremely close to each other.

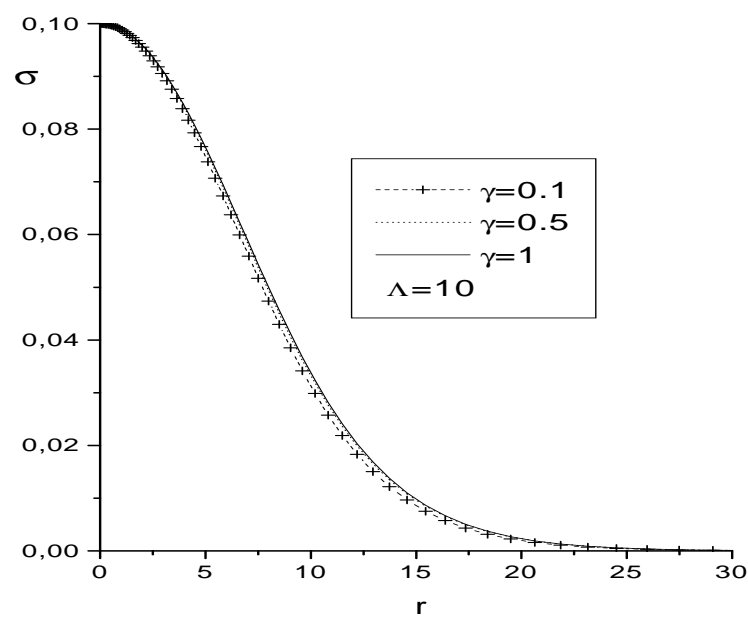

FIG. 4. The dependence $\sigma(r)$ for three different values of the parameter $\gamma$.

The dependence $\tilde{\nu}(r)$ is shown in Fig. 5 for three different values of $\gamma$. As it is seen the influence of $\gamma$ on $\tilde{\nu}(r)$ is slight.

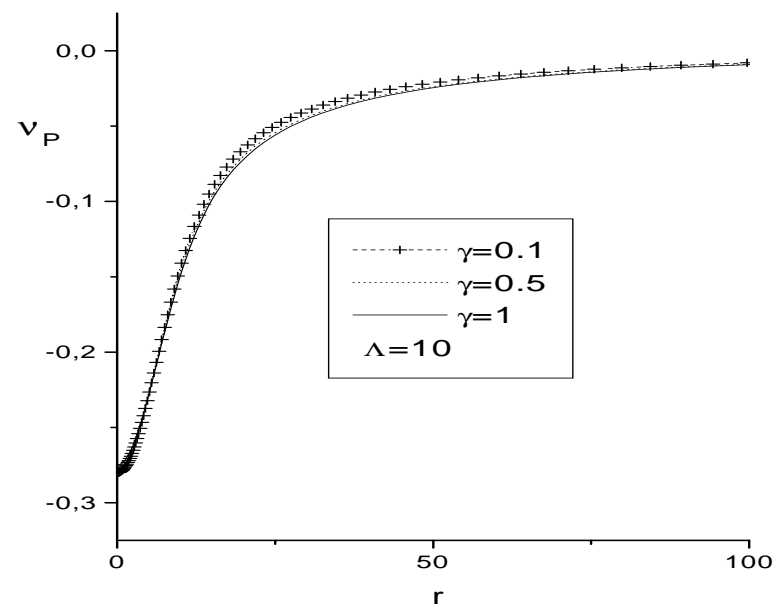

FIG. 5. The physical function $\tilde{\nu}(r)$ for three different values of the parameter $\gamma$.

The most sensitive to the value of the parameter $\gamma$ is the dilaton field $\varphi$. The dependence $\varphi(r)$ is presented in Fig. 6 for three different values of $\gamma$. One sees that the increasing of $\gamma$ leads to suppressing the dilaton field as one must expect. The dependence $\Phi(r)$ is shown in Fig. 7. At the center of the star one has $\Phi(0)>1$ which means that the gravitational constant at the center satisfies $G(0)<1$. Therefore the boson stars are less 


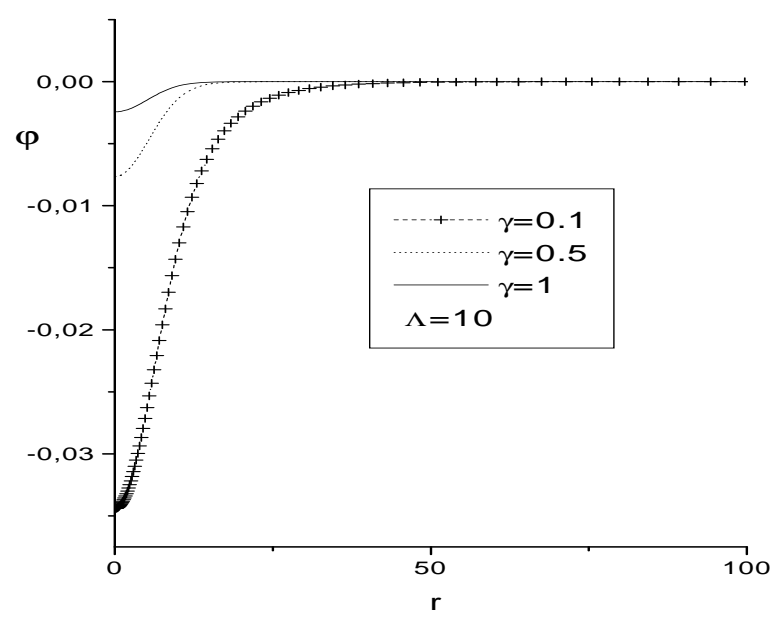

FIG. 6. The dependence $\varphi(r)$ for three different values of the parameter $\gamma$.

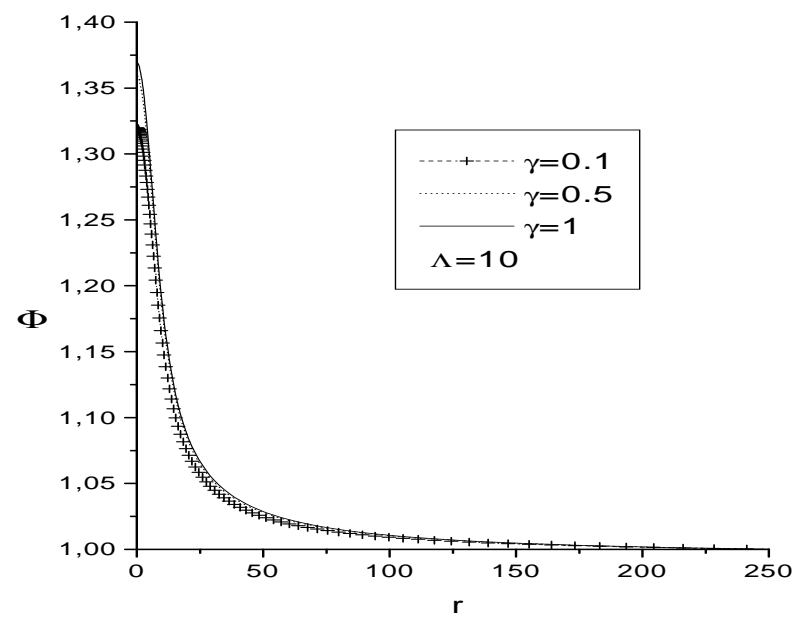

FIG. 7. The function $\Phi(r)$ for three different values of the parameter $\gamma$

gravitationally bounded than in general relativistic case.

We have also examined the cases of different values of $\Lambda$. The picture is qualitatively the same.

It is interesting to know how the exact form of the dilaton potential $V(\varphi)$ influences the boson star structure. We have examined several potentials $V(\varphi)$ with the same dilaton mass (for example $V(\varphi)=2 \varphi^{2}, V(\varphi)=2 \sin ^{2}(\varphi)$, $\left.V(\varphi)=4(1-\cos (\varphi)), V(\varphi)=2\left(1-\exp \left(-\varphi^{2}\right)\right)\right)$. Our numerical results show that boson star structure in practice does not depend on the exact form of the dilaton potential in the class of potential we have examined. This gives strong evidences that the boson star structure is sensitive mainly to the second derivative of the dilaton potential $\frac{d^{2} V(\varphi)}{d \varphi^{2}}(0)$ determining the dilaton mass rather to the exact form of the potential.

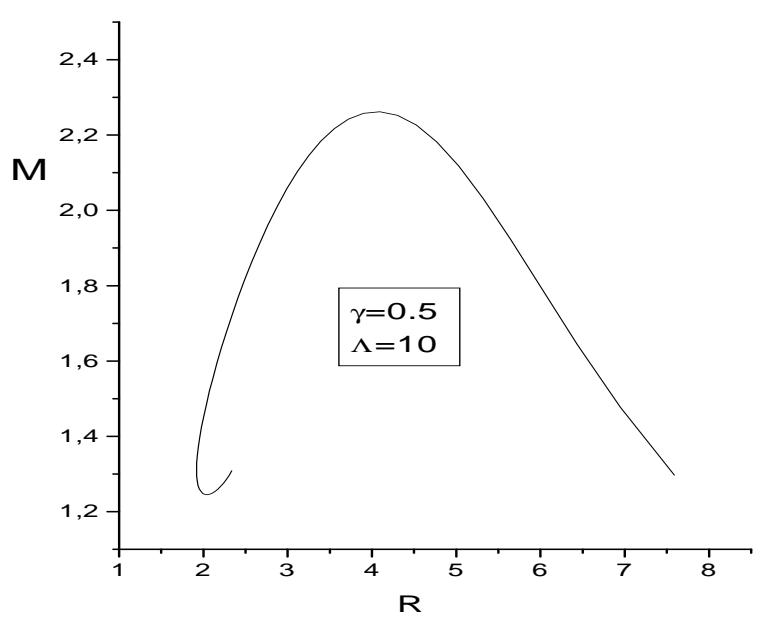

FIG. 8. The boson star mass $M$ as a function of the boson star radius $R$

Finally in Fig. 8 one can see that the usual dependence of the mass $M(R)$ of boson star on its effective radius $R$ take place in the scalar-tensor model with massive dilaton, too. As seen, there exist a domain of stability of the boson star, followed by domains of unstable states.

\section{BOSON STAR STABILITY}

Here we briefly discuss stability property of the boson stars we consider. Our analysis is based on catastrophe theory 27.

The basis for our analysis is Fig. 9 where the binding energy $E_{b}$ is plotted against the rest mass $M_{R}$ (i.e. particle number). This figure, as our numerical results show, is typical for a large number of values of $\gamma$ and $\Lambda$. Therefore the forthcoming conclusions concern the general case not only the special case presented by the figure.

Fig. 9 is actually a bifurcation diagram. For small central densities the binding energy is negative which shows that the boson star is potentially stable and one assumes that the boson star is stable against small radial perturbations. As the central density is increased one meets a cusp. The second branch as a whole has higher mass and therefore it is unstable. At the cusp, the boson star stability changes - one radial perturbation mode develops instability. 


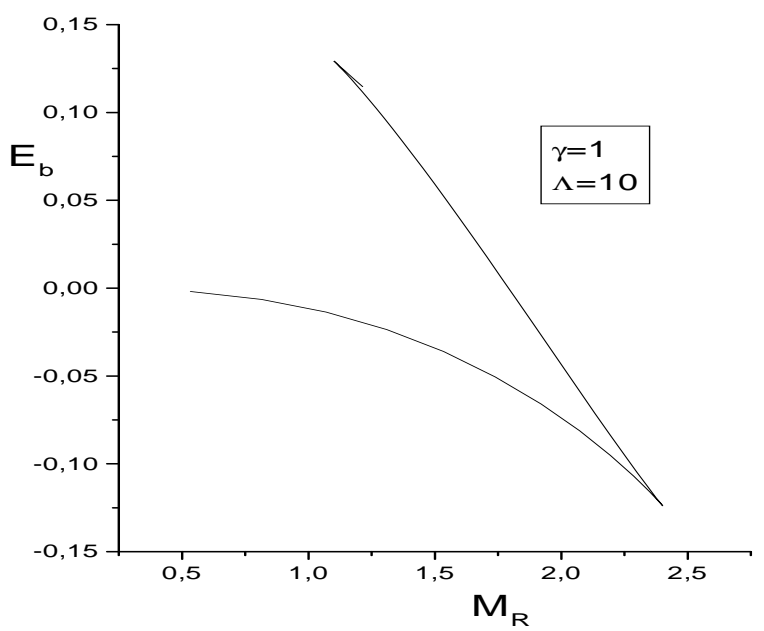

FIG. 9. The boson star binding energy $E_{b}$ as a function of the rest mass $M_{R}$

\section{CONCLUSION}

In this paper we have analyzed static spherically symmetric boson stars in a scalar tensor theory of gravity with massive gravitational scalar (dilaton). We have studied their equilibrium properties for different values of the ratio $\gamma=\frac{m}{m_{B}}$. The conclusion is that stable boson stars may exist for any value of $\gamma$. When $\gamma$ increases the equilibrium configurations tends to those from general relativity and for sufficiently large $\gamma$ the general relativistic case is in practice recovered. The masses of the boson stars are turned out to be always smaller than those in general relativity. The typical deviations of the masses in our model of dilatonic gravity from those in general relativity is a few percent. The small deviations of bosonic star structure in the considered model of dilatonic gravity from general relativistic case are significantly greater then the corresponding deviations in solar system and Earth surface experiments studied in [18]. It turns out also that the boson star structure is sensitive mainly to the mass term of the dilaton potential rather to the exact form of the potential. The boson stars in our case are less gravitationally bound as a consequence of the fact that the gravitational constant $G$ within the stars is smaller than one.

For completeness we have examined also the case of scalar-tensor theories with a massive gravitational scalar with kinetic term $\omega_{B D} \Phi^{-2} \tilde{g}^{\mu \nu} \partial_{\mu} \Phi \partial_{\nu} \Phi$. In these cases the obtained results for different values of the parameter $\omega_{B D}$ are qualitatively the same as in the case $\omega_{B D} \equiv 0$ and for large $\omega_{B D}$ the differences between bosonic stars in dilatonic gravity and in general relativity are even smaller.

It is worth noting that, in the domain of stability of boson stars, our results are qualitatively close to the results obtained for boson stars in Brans-Dicke theory [ 4 , [5], and for boson stars considered in a scalar-tensor theory with a kinetic term and simple coupling between the dilaton and the mass term of the boson filed in Jordan frame [6] which in our notations reads $m_{B}^{2} \Phi \Psi^{+} \Psi$. In the domain of instability of boson stars the results of the last reference differ essentially from ours. As in general relativity and Brans-Dicke theory beyond the point of stability for large central densities the boson star mass in our model of massive dilatonic gravity drops, oscillates a bit and approaches a constant value while in the model considered in [6] it increases rapidly.

Acknowledgments: We are grateful to the unknown referee for useful suggestions and for pointing out the references [6] and [12]. This work was supported by Bulgarian National Scientific Fund, Contr. NoNo F610/99, MM602/96 and by Sofia University Research Fund, Contr. No. 245/99.

[1] D. Kaup, Phys. Rev. 172, 1331 (1968)

[2] R. Ruffini, S. Bonazzola, Phys. Rev. 187, 1767 (1969)

[3] M. Colpi, S. Shapiro, I. Wasserman, Phys. Rev. Lett. 57, 2485 (1986)

[4] M. Gunderson, L. Jensen, Phys. Rev. DD48, 5628 (1993)

[5] D. Torres, Phys. Rev. DD56, 3478 (1997)

[6] Z. Tao, X. Xue, Phys. Rev. D45, 1878 (1992)

[7] Torres D, Liddle A, Schunck F, Phys. Rev. D57, 4821 (1998)

[8] G. Comer, H. Shinkai, Class. Quantum Grav. 15, 669 (1998)

[9] D. Torres, F. Schunck, A. Liddle, Class. Quantum Grav. 15, 3701 (1998)

[10] J. Barrow, Phys. Rev. D46, 3227 (1992)

[11] J. Balakrishna,H. Shinkai, Phys. Rev. D58, 044016-1 (1998)

[12] A. Whinnett, D. Torres, Phys. Rev. D60, 104050, (1999)

[13] A. A. Starobincky, JETP Lett. 68, 757 (1998); astroph/9811360; V. Sahni, A. A. Starobinsky, astroph/9904398; T. D. Saini, S. Raychaudhury, V. Sahni, A. A. Starobinsky, astro-ph/9910231; B. Boisseau, G. Esposito-Farése, D. Polarski, A. A. Starobinsky, grqc/0001066.

[14] J. O'Hanlon, Phys. Rev. Lett. 29, 137 (1972).

[15] Y. Fujii, Nature (London) Phys. Sci, 234, 5 (1971); Ann. Phys. (NY) 69, 494 (1972). J. O'Hanlon, Phys. Rev. Lett. 29, 137 (1972). Y. Fujii, Phys. Rev. D 9, 874 (1974).

[16] Y. Fujii, Gravitation and scalar field, Kodan-sha, Tokyo, 1997.

[17] P. Fiziev, Torsion Dilaton and Novel Minimal Coupling Principle, E-print: gr-qc/9809001;

[18] P. Fiziev, A Realistic Model of Dilatonic Gravity, E-print: gr-qc/9911037. 
[19] T. Helbig, ApJ 382, 223 (1991).

[20] A. De Rújula, Phys. Lett. B 180, 213 (1986).

[21] C. Talmage, in Proc. Sixth Marcel Grossman Meeting on General Relativity, ed. H. Sato and T. Nakamura, World Scientific, Singapore (1992)

[22] A. Whinnett, Class. Quant. Grav. 16, 2796 (1999)

[23] S. Yazadjiev , Class. Quantum Grav. 16, L63 - L69 (1999)

[24] M. Gavurin, Izvestia VUZ, Matematika,No 5, pp. 18-31, (1958) (in Russian).

[25] E. Jidkov et al., In "Physics of Elementary particles and atomic Nuclei", vol. 4, Part. 1 (1973), JINR, Dubna (in Russian).

[26] T. Boyadjiev, M. Todorov, P. Fiziev, S. Yazadjiev, Mathematical Modeling of Boson-Fermion Stars in the Generalized Scalar-Tensor Theories of Gravity, E-print: math.SC/9911118

[27] F. Kusmartsev, E. Mielke, F. Shunk, Phys. Rev. D 43, 3895 (1991) 